Original Paper http://ajol.info/index.php/ijbcs http://indexmedicus.afro.who.int

\title{
Caractérisation des peuplements ligneux sur le tracé de la Grande Muraille Verte au Tchad
}

\author{
Minda MAHAMAT-SALEH ${ }^{1}$, Ousmane NDIAYE ${ }^{1}$, Mariama Dalanda DIALLO ${ }^{2}$, \\ Saradoum GOY ${ }^{1}$, Khoudia NIANG ${ }^{1}$, Aly DIALLO ${ }^{1}$, Aliou GUISSE ${ }^{1,3 *}$ \\ ${ }^{1}$ Département Biologie Végétale, Faculté des Sciences et Techniques, \\ Université Cheikh Anta Diop de Dakar, B.P 5005 Dakar, Sénégal. \\ ${ }^{2}$ Section Productions Végétales et Agronomie, UFR des Sciences Agronomiques, de l'Aquaculture et des \\ Technologies Alimentaires, Université Gaston Berger de Saint Louis, B.P 234 Saint Louis, Sénégal. \\ ${ }^{3}$ Observatoire Homme Milieu Tessekere, UMI 3189 \\ *Auteur correspondant ; E-mail: alguisse@orange.sn, Tel. : (+221) 776389669
}

\section{REMERCIEMENTS}

Ce travail a été réalisé grâce à l'appui financier de l'Agence Panafricaine de la Grande Muraille Verte et de l'Agence Nationale de la Grande Muraille du Tchad.

\section{RESUME}

La connaissance des caractéristiques du peuplement ligneux du tracé de la Grande Muraille Verte (GMV) du Tchad permet de comprendre leur structure et fonctionnement afin de proposer des stratégies de gestion durable. C'est dans ce cadre que ce travail vise à caractériser la flore et la végétation ligneuses sur le tracé de la GMV du Tchad. La méthodologie utilisée consiste à déterminer la composition floristique et la structure du peuplement ligneux dans les trois sites retenus (Lac, Kanem et Bahr El Ghazal). Les inventaires floristiques ont permis de recenser 18 espèces réparties en 15 genres et 9 familles. La flore ligneuse est dominée par la famille des Mimosaseae. La densité des ligneux (34,67 ind.ha $\left.{ }^{-1}\right)$ et le recouvrement (1604,30 $\mathrm{m}^{2}$.ha ${ }^{-1}$ ) sont plus importants au Lac et faible au Bahr El Ghazal (densité de 21,39 ind.ha ${ }^{-1}$ et recouvrement de $\left.465,66 \mathrm{~m}^{2} \cdot \mathrm{ha}^{-1}\right)$, quant à la surface terrière, elle est plus élevée au Kanem $\left(3,39 \mathrm{~m}^{2} \cdot \mathrm{ha}^{-1}\right)$. La distribution par classes de hauteur et de circonférence des ligneux révèle une prédominance des individus de la strate arbustive dans les trois sites. L'étude de la régénération naturelle montre que Acacia raddiana et Balanites aegyptiaca présentent le potentiel de régénération le plus élevé dans les trois sites. Globalement, les résultats obtenus montrent que les espèces les plus adaptées aux conditions écologiques de la GMV au Tchad sont Acacia raddiana et Balanites aegyptiaca et par conséquent, doivent être choisies pour la restauration de ces écosystèmes.

(c) 2015 International Formulae Group. All rights reserved.

Mots clés : Tchad, GMV, végétation, peuplement, structure, régénération.

\section{Characterization of the ligneous populatings on the route of the Great Green Wall in Chad}

\begin{abstract}
The knowledge of ligneous populating characteristics of the route of the Great Green Wall (GGW) of Chad helps to understand their structure and function in order to propose strategies for sustainable management. This study was carried out to characterize the flora and the tree vegetation present on the Chad
\end{abstract}


Great Green Wall (GGW) line. The methodology consisted in determining the floristic composition and the structure of the tree population in three sites: Lac, Kanem, and Bahr El Ghazal. The floristic inventories have identified 18 species distributed into 15 genera and 9 families. Trees are dominated by the Mimosaseae family. Their density (34.67 individuals ha ${ }^{-1}$ ) and their recovery $\left(1604.3 \mathrm{~m}^{2} \mathrm{ha}^{-1}\right)$ are more important at Lac and lower at Bahr El Ghazal with 21.39 individuals $\mathrm{ha}^{-1}$ and $465.66 \mathrm{~m}^{2} \mathrm{ha}^{-1}$, respectively. However, the basal area of the vegetation was more important at Kanem with $3.39 \mathrm{~m}^{2} \mathrm{ha}^{-1}$. The distribution by classes of height and circumference of the trees indicates a predominance of the shrub layer in all the three sites. The study of the natural regeneration reveals that Acacia raddiana and Balanites aegyptiaca present the highest regeneration potential in the three sites. Globally, the results obtained in this study show that the most adapted species to the ecological conditions of the Chad's GGW are Acacia raddiana and Balanites aegyptiaca. Consequently, these species must be considered during the restoration of the given ecosystems.

(C) 2015 International Formulae Group. All rights reserved.

Keywords: Chad, GGW, vegetation, population, structure, regeneration.

\section{INTRODUCTION}

La dégradation des sols est un phénomène très marqué dans les pays africains de la zone sahélo-saharienne. Elle est accentuée par les variabilités climatiques dont les manifestations sont les sécheresses (Ndiaye et al., 2014). Cette dégradation des terres qui fait suite à la désertification liée au déficit pluviométrique et/ou aux activités anthropiques réduit énormément le potentiel biologique des sols (Boubacar, 2010) provoquant une baisse de la production agricole et des ressources naturelles. Pour faire face à cette situation, les pays du Sahel ont initié un grand projet dénommé Grande Muraille Verte (GMV), une stratégie pour lutter contre la désertification et réhabiliter les sols dégradés. Cette GMV est une ceinture de végétation plurispécifique, sur une longueur d'environ $7000 \mathrm{~km}$ et une largeur de $15 \mathrm{~km}$ et qui englobe onze pays africains (Sénégal, Burkina Faso, Djibouti, Erythrée, Ethiopie, Mali, Mauritanie, Niger, Nigeria, Soudan et Tchad) sur l'axe Dakar-Djibouti (GMV, 2009).

Comme pour le reste des pays de l'Afrique sahélienne, la zone aride et semiaride du Tchad est marquée par la rareté de la strate arborée (Ngaryo et al., 2010), cependant, cette zone subit depuis plusieurs décennies de fortes perturbations liées d'une part aux conditions naturelles d'aridité (longue saison sèche, forte évaporation, des faibles précipitations) et d'autre part à une surexploitation extensives des ressources qui accentuent la péjoration des conditions climatiques (Ndiaye et al., 2014; SNPA/GMV, 2012). La connaissance des caractéristiques du peuplement végétal permet de mieux comprendre les écosystèmes de cette zone, de les décrire dans leurs aspects afin de proposer des stratégies de gestion durable. La présente étude se propose de mettre en évidence l'importance de la diversité floristique ligneuse, de caractériser la végétation ligneuse en déterminant la densité, le recouvrement, la surface terrière et évaluer la régénération naturelle du peuplement ligneux des espèces dans les trois sites du tracé de Grande Muraille Verte du Tchad.

\section{MATERIEL ET METHODES \\ La zone d'étude}

La zone d'étude s'étend sur $455 \mathrm{~km}$ de long et $15 \mathrm{~km}$ de large, elle fait partie du tracé de la GMV du Tchad (Figure 1). Le climat est de type sahélien caractérisé par deux saisons: une saison sèche qui s'étend de novembre à mai et une saison pluvieuse de juin à octobre. La température varie de 22,31 ${ }^{\circ} \mathrm{C}$ à 33,64 ${ }^{\circ} \mathrm{C}$ (ASECNA, 2012). Trois sites (Lac, Kanem et Bahr El ghazal) ont été utilisés pour la caractérisation de la zone d'étude. Leur choix a été motivé par les différentes unités pédologiques rencontrées. Le Lac, situé à l'Ouest du tracé, est caractérisé par les sols sableux et par Acacia raddiana et Balanites aegyptiaca (Pias, 1970; Mahamat-Saleh et al., 2012) comme espèces dominantes. Le Kanem, situé au Centre, est caractérisé par les sols argileux, à concrétion 
calcaire et par l'abondance de Acacia raddiana et par Hyphaene thebaica (Mahamat-Saleh et al., 2012). Le Bahr El Ghazal, situé à l'Est du tracé, est caractérisé par des sols argilo-sableux et par Balanites aegyptiaca comme espèce dominante.

\section{Collecte des données}

L'échantillonnage a consisté à répartir de façon aléatoire comme suggéré par Niang et al. (2014). 71 placettes de $50 \mathrm{~m}$ x 50 $\mathrm{m}\left(2500 \mathrm{~m}^{2}\right)$ de surface chacune dans chaque site en fonction des différentes unités morphopédologiques (dune, plateau et dépression) : 12 placettes au Lac, 36 au Kanem et 23 placettes au Bahr El Ghazal. Dans chaque placette, la liste des espèces a été établie. Des mesures dendrométriques ont été effectuées. Les mensurations ont concerné les individus à circonférence basale supérieure ou égale à $10 \mathrm{~cm}$ (Diallo et al., 2011). Pour chaque individu rencontré, les mesures ont porté sur la circonférence à la base du tronc à $30 \mathrm{~cm} \mathrm{du}$ sol, le diamètre de la projection du houppier au sol dans deux directions (NordSud et Est-Ouest), la hauteur des arbres et la distance entre deux arbres. Ces mesures ont été cumulées au comptage de la régénération $\mathrm{du}$ peuplement. Les données recueillies ont été gérées et traitées avec le tableur Excel et le logiciel Minitab afin d'évaluer les paramètres de structure (densité, surface terrière, surface de couronne, distribution). L'inventaire et l'analyse floristique ont permis de dresser la liste des espèces recensées et de les regrouper par famille.

\section{Traitements des données}

Les données suivantes ont été déterminées :

- la densité réelle qui est le nombre d'individus par unité de surface (individus.ha $^{-1}$ );

- la densité théorique qui peut être exprimée par l'écartement moyen entre les individus en utilisant la méthode du plus proche individu. Son calcul se fait par le rapport de la surface d'un hectare $\left(10000 \mathrm{~m}^{2}\right)$ sur le carré de la distance moyenne entre les arbres (dm) (Wouters et Notelaers, 1999).

$$
\text { Densité théorique }=\frac{10000}{(\mathrm{dm})^{2}}
$$

$\mathrm{dm}$ : représente la distance moyenne entre les individus $(\mathrm{m})$;

10000 : c'est la surface d'un carré de 100 m de côté $=10000 \mathrm{~m}^{2}=1$ ha.

- la surface terrière (St) ou recouvrement basal, est la somme des surfaces terrières de tous les individus dont la circonférence basale (C) est supérieure ou égale à $10 \mathrm{~cm}$. Cette surface terrière peut être estimée en considérant que les sections des tiges sont circulaires. Elle s'exprime par unité de surface $\left(\mathrm{m}^{2} \cdot \mathrm{ha}^{-1}\right)$ et se calcule tel que décrit par Ndiaye et al. (2014).

$$
\mathrm{St}=\sum \frac{\mathrm{C}^{2}}{4 \pi}
$$

$\mathrm{St}=$ surface terrière exprimée en $\mathrm{m}^{2} \cdot \mathrm{ha}^{-1} ; \mathrm{C}=$ circonférence à $30 \mathrm{~cm}$ du sol des individus mesurée en centimètre.

- le recouvrement aérien $(\mathrm{Sc})$ est la somme des surfaces de la couronne de tous les individus du peuplement. Elle est obtenue à l'aide du diamètre moyen du feuillage de l'arbre (D) assimilé à un cercle par projection sur le sol. Elle est obtenue par la formule suivante (Diallo et al., 2011).

$$
\mathrm{Sc}=\sum \frac{\pi \times \mathrm{D}^{2}}{4}
$$

$\mathrm{S}_{\mathrm{C}}=$ surface de la couronne exprimée en $\mathrm{m}^{2} \cdot \mathrm{ha}^{-1} ; \mathrm{D}=$ moyenne des diamètres Est/Ouest et Nord/Sud exprimée en mètre.

La répartition des individus en fonction des circonférences et hauteurs a permis d'évaluer les structures horizontale et verticale du peuplement (arbustif ou arboré). Ndiaye et al. (2014) appelle «arbustes » les individus adultes dont la hauteur ne dépasse pas $7 \mathrm{~m}$ et « arbres » ceux dont la hauteur dépasse $7 \mathrm{~m}$.

- le taux de régénération du peuplement est donné par le rapport en pourcentage entre l'effectif total des jeunes plants (circonférence $<10 \mathrm{~cm}$ ) et l'effectif total du peuplement. 


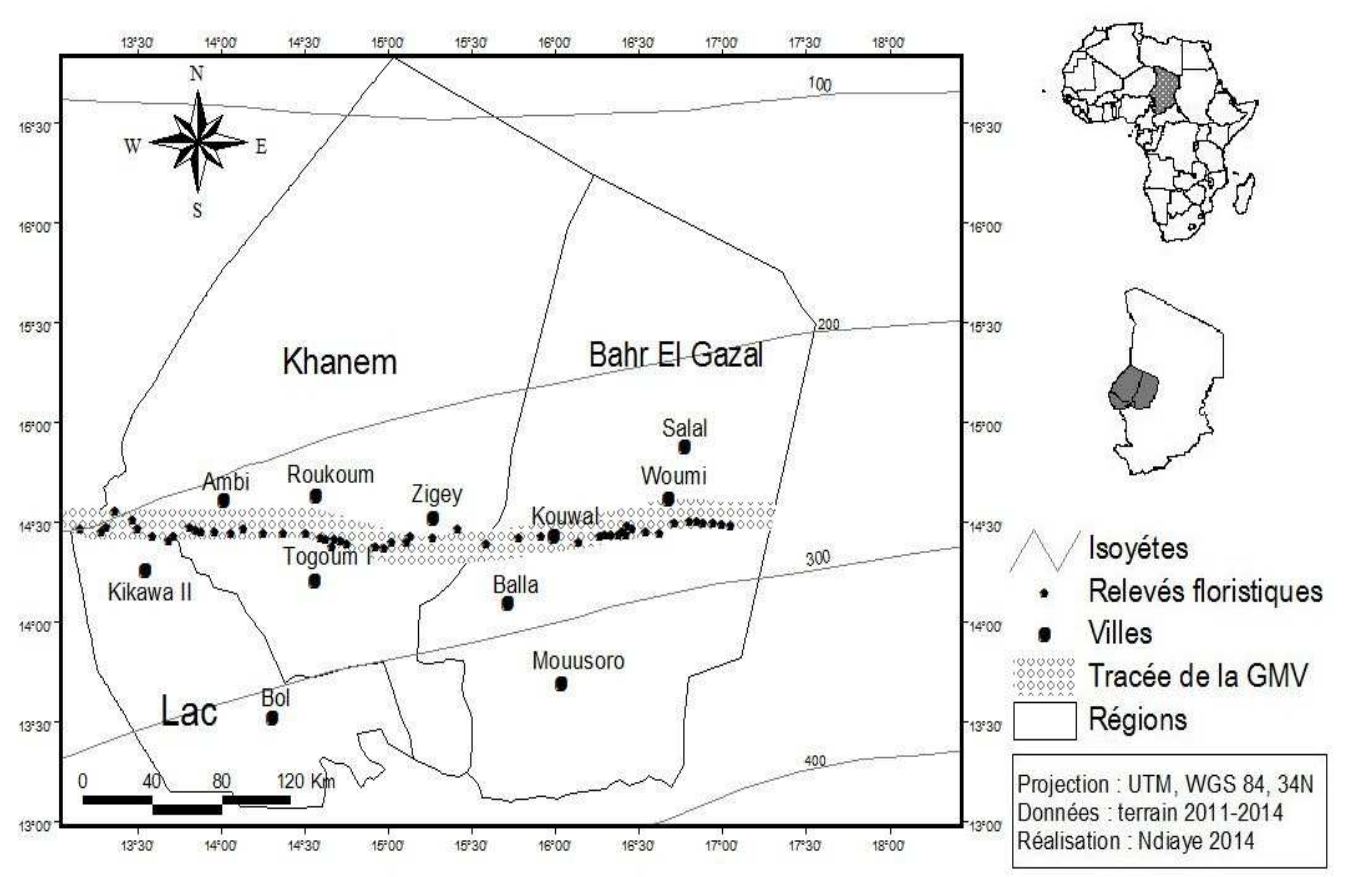

Figure 1 : Situation des sites d'étude.

\section{RESULTATS}

\section{Diversité floristique}

Au total, 18 espèces ont été recensées. Ces espèces sont réparties en 15 genres et 9 familles. Les familles les plus représentées sont les Mimosaseae (4 espèces), les Capparaceae et les Apocynaceae ( 3 espèces), les Rhamnaceae ( 2 espèces) et les Arecaceae ( 2 espèces) (Tableau 1). La flore est riche de 5 familles au Lac (Balanitaceae, Capparaceae, Apocynaceae, Mimosaseae, Salvadoraceae) 9 au Kanem (Balanitaceae, Capparaceae, Rhamnaceae, Apocynaceae, Burseraceae, Moraceae, Arecaceae, Mimosaseae, Salvadoraceae) et 6 au Bahr El Ghazal (Balanitaceae, Capparaceae, Rhamnaceae, Apocynaceae, Mimosaseae, Salvadoraceae).

\section{Répartition de la flore en fonction des sites}

La composition de la flore varie suivant les sites. Au Kanem, 17 espèces reparties en 14 genres et 9 familles ont été inventoriées. La famille des Mimosaseae est plus représentée (3 genres et 4 espèces), suivie des Capparaceae (3 genres et 3 espèces). Bahr El Ghazal présente 10 espèces, 7 genres et 6 familles. Les Capparaceae sont les plus représentées (3 genres et 3 espèces), suivies des Apocynaceae et des Mimosaseae (chacune 1 genre et 2 espèces). Au Lac, nous avons inventorié 8 espèces reparties en 7 genres et 5 familles. Les Apocynaceae sont les mieux représentées ( 2 genres et 3 espèces), suivie des Mimosaseae ( 2 genres et 2 espèces). (Tableau 1).

\section{Structure du peuplement ligneux}

La densité réelle et la densité théorique, la surface terrière et le recouvrement des différents sites sont consignées dans le Tableau 2. 
Dans la zone d'étude, la densité

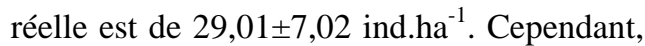
elle est plus élevée au Lac $(34,67 \pm 5,44$ ind.ha $\left.{ }^{-1}\right)$ que dans le Kanem (32 $\pm 6,22$ ind.ha ${ }^{-}$ $\left.{ }^{1}\right)$ et le Bahr El Ghazal $\left(21,39 \pm 3,02\right.$ ind.ha $\left.^{-1}\right)$.

Le taux de recouvrement est de $1160,81 \pm 4,87 \mathrm{~m}^{2} \cdot \mathrm{ha}^{-1}$ dans l'ensemble de la zone d'étude. Il varie en fonction des différents sites. Il est plus élevé au Lac $\left(1604,30 \pm 159,41 \mathrm{~m}^{2} \cdot \mathrm{ha}^{-1}\right)$, moyen au Kanem $\left(1457,12 \pm 61,94 \mathrm{~m}^{2} \cdot \mathrm{ha}^{-1}\right)$ et faible au Bahr El Ghazal $\left(465,66 \pm 24,53 \mathrm{~m}^{2} \cdot \mathrm{ha}^{-1}\right)$.

La surface terrière de la zone d'étude est de 2,49 $\pm 0,01 \mathrm{~m}^{2} \cdot \mathrm{ha}^{-1}$. Elle est plus élevée au Kanem $\left(3,39 \pm 0,03 \mathrm{~m}^{2} \cdot \mathrm{ha}^{-1}\right)$, moyenne au Lac $\left(2,27 \pm 0,02 \mathrm{~m}^{2} \cdot \mathrm{ha}^{-1}\right)$ et faible au Bahr El Ghazal $\left(1,21 \pm 0,01 \mathrm{~m}^{2} \cdot \mathrm{ha}^{-1}\right)$.

\section{Distribution des peuplements}

\section{Distribution des individus selon la hauteur}

La Figure 2 montre la distribution de Weibull à trois paramètres du peuplement ligneux et des espèces dominantes selon des classes de hauteur dans les trois sites (Lac, Kanem et Bahr El Ghazal). Cette Figure 2 indique une prédominance des individus dans les premières classes de hauteur. Le paramètre de forme est inférieur à 2,563 dans tous les sites avec un coefficient de forme compris entre 1 et 3 .

La hauteur des individus $\mathrm{du}$ peuplement du Lac varie entre 0 et $18 \mathrm{~m}$, celle de la population de Acacia raddiana entre 0 et $24 \mathrm{~m}$ et les Balanites aegyptiaca entre 0 et $12 \mathrm{~m}$. Les individus du peuplement présentent une distribution en «J » renversée (paramètre de forme $<1$ ) indiquant la prédominance d'individus dont la hauteur est comprise entre 1 et $18 \mathrm{~m}$. Au Kanem, les individus du peuplement et les espèces dominantes indiquent une prédominance d'individus dont la hauteur est comprise entre 0 et $18 \mathrm{~m}$. Les individus de la population de Acacia raddiana sont présents dans toutes les classes de hauteur avec une prédominance dans les classes inferieures (1 à $6 \mathrm{~m}$ ). C'est dans les classes comprises entre 6 et $9 \mathrm{~m}$ que la plupart des individus du Balanites aegyptiaca se regroupent. Au Bahr El Ghazal, la distribution selon la hauteur des individus du peuplement varie de 0 à $18 \mathrm{~m}$, pour Acacia raddiana, elle varie de 0 et $21 \mathrm{~m}$ et pour Balanites aegyptiaca de 0 à $18 \mathrm{~m}$. Dans ce site, le peuplement et les espèces dominantes présentent une distribution asymétrique droite. La distribution des individus $\mathrm{du}$ peuplement montre une dominance des sujets dont la hauteur est comprise entre 0 et $9 \mathrm{~m}$. En ce qui concerne la population de Acacia raddiana, elle regroupe les individus dont la hauteur est comprise entre 0 et $15 \mathrm{~m}$, avec une bonne représentation des individus dans la classe 9 $\mathrm{m}$.

Distribution des individus selon la circonférence

La Figure 3 montre la distribution de Weibull à trois paramètres du peuplement ligneux et des espèces dominantes selon des classes de circonférence dans les trois sites (Lac, Kanem et Bahr El Ghazal).

L'analyse de la figure 3 montre une distribution asymétrique droite (paramètre de forme compris entre 1 et 3,6 ; elle montre aussi une prédominance des individus dans les premières classes de circonférence avec un coefficient de forme compris entre 1 et 3 $\mathrm{m}$.

La circonférence des individus du peuplement du Lac et celle de la population de Balanites aegyptiaca varient entre 10 et $300 \mathrm{~cm}$ et alors que celle de la population de Acacia raddiana est comprise entre 10 et 200 $\mathrm{cm}$. Concernant le Kanem, la circonférence $\mathrm{du}$ tronc des individus du peuplement varie de 10 à $300 \mathrm{~cm}$ et celle des espèces dominantes entre 10 à $200 \mathrm{~cm}$. Ce site est caractérisé par la dominance des individus 
de petites classes de circonférence $(50 \mathrm{~cm})$. $\mathrm{Au}$ Bahr El Ghazal, les individus dont la circonférence est inférieure à $200 \mathrm{~cm}$ sont très peu représentés. Dans ce site, les individus ayant une circonférence comprise entre 50 et $100 \mathrm{~cm}$ sont prépondérants.

La distribution du peuplement ligneux selon la hauteur et la grosseur montre que la végétation est dominée par des arbustes, donc il s'agit de savanes arbustives.

\section{Régénération du peuplement ligneux}

L'importance de la régénération en fonction des espèces est représentée par la Figure 4.

En tenant compte des espèces, Acacia raddiana $(59,02 \%)$ a le plus fort taux de régénération, suivie de Balanites aegyptiaca $(17,66 \%)$ et de Hyphaene thebaica $(9,69 \%)$. Les espèces dominantes ont les taux de régénération les plus élevés $(86,37 \%)$. Les autres espèces ont une faible régénération.

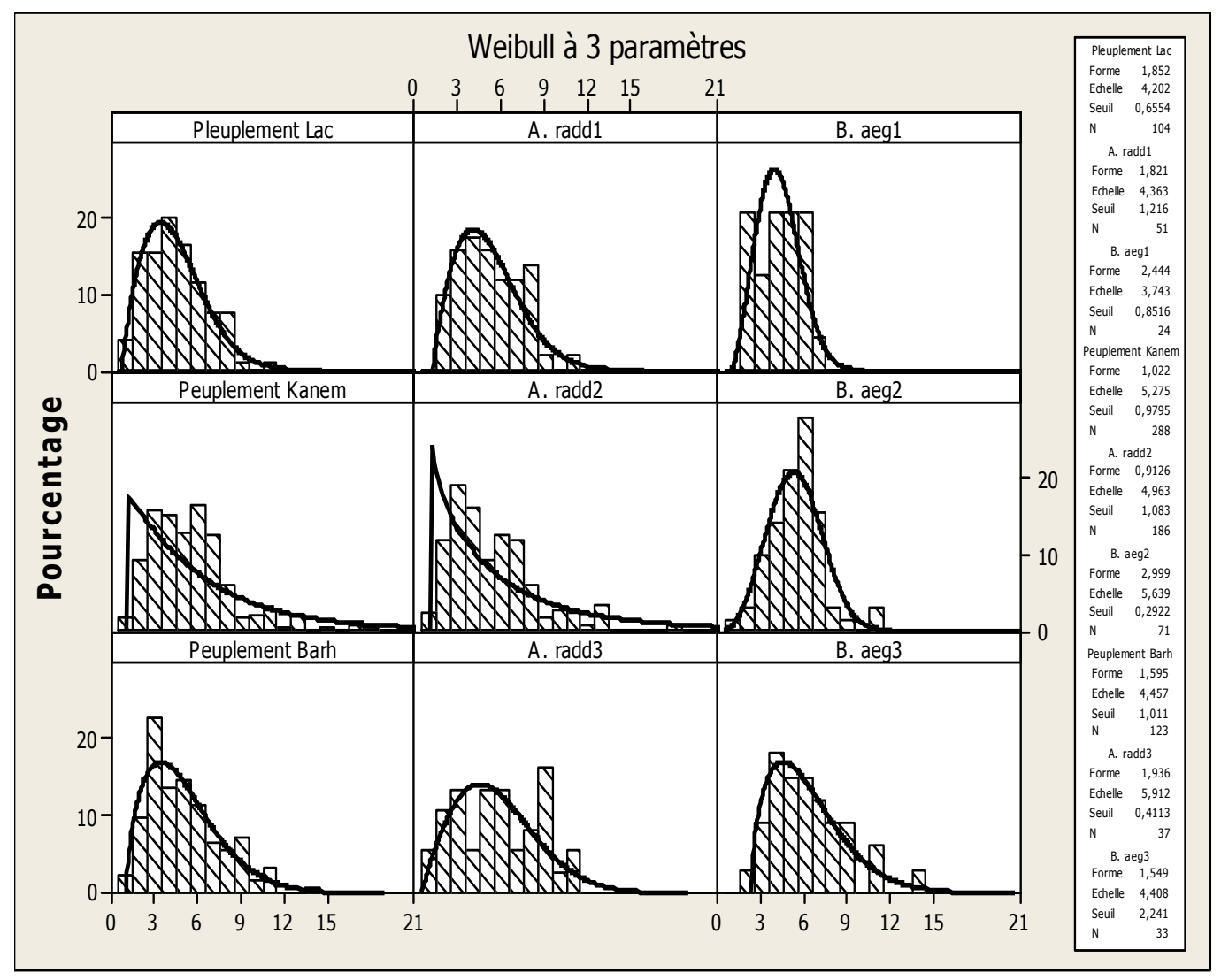

Figure 2 : Distribution des individus du peuplement et des espèces dominantes par centre de classe de hauteur (A. radd : Acacia raddiana, B. aeg : Balanites aegyptiaca). 


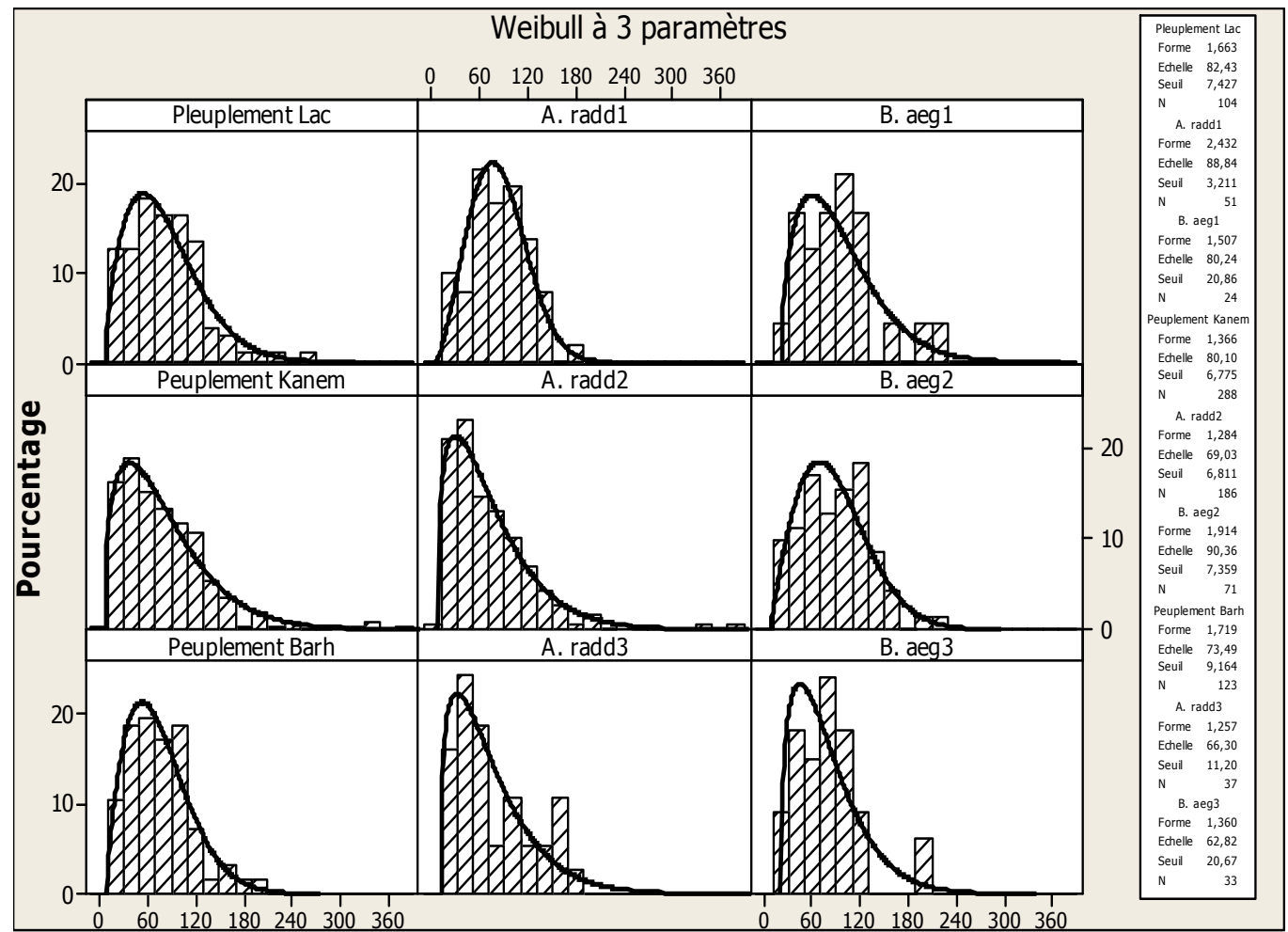

Figure 3 : Distribution du peuplement et des espèces dominantes par centre de classe de circonférence à $30 \mathrm{~cm}$ du sol (A. radd : Acacia raddiana, B. aeg : Balanites aegyptiaca).

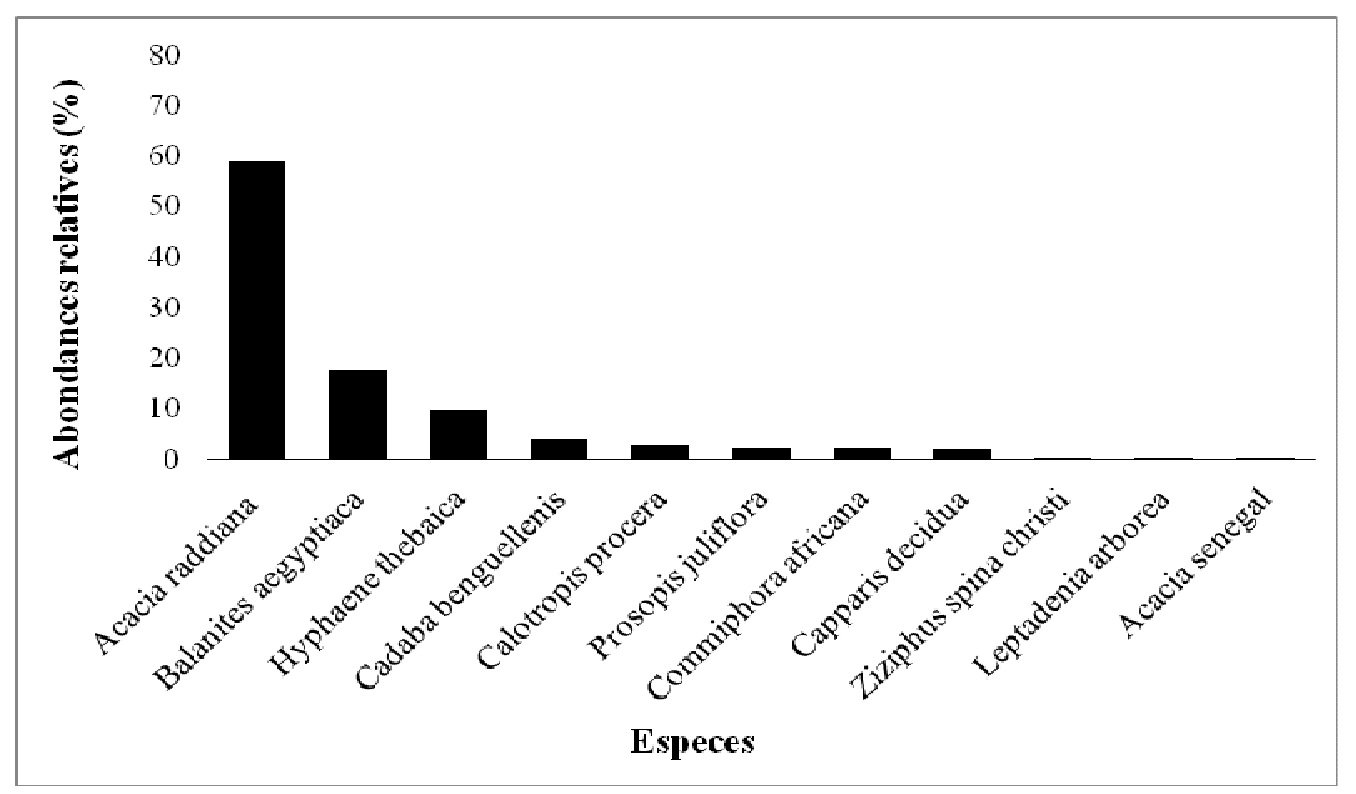

Figure 4 : Nombre de rejets par espèce de la zone d'étude. 
Tableau 1 : La flore inventoriée en fonction des différents sites.

\begin{tabular}{|c|c|c|c|c|}
\hline \multirow[b]{2}{*}{ Familles } & \multirow[b]{2}{*}{ Espèces } & \multicolumn{3}{|c|}{ Sites } \\
\hline & & 1 & 2 & 3 \\
\hline Balanitaceae & Balanites aegyptiaca (L.) Del & $*$ & $*$ & $*$ \\
\hline \multirow{3}{*}{ Capparaceae } & Capparis decidua (Forssk.) Edgew & $*$ & $*$ & $*$ \\
\hline & Cadaba benguellenis Mendes & & $*$ & * \\
\hline & Boscia senegalensis (Pers.) Lam. & & $*$ & $*$ \\
\hline \multirow{2}{*}{ Rhamnaceae } & Ziziphus abyssinica A.Rich & & $*$ & \\
\hline & Ziziphus spina christi (L) Desf. & & $*$ & * \\
\hline \multirow{3}{*}{ Apocynaceae } & $\begin{array}{l}\text { Calotropis procera (Aiton) W.T.Aiton } \\
\text { Leptadenia pvrotechnica (Forsk.) }\end{array}$ & $*$ & & \\
\hline & Decne & $*$ & $*$ & $*$ \\
\hline & Leptadenia arborea (Forssk) Schwein & $*$ & $*$ & $*$ \\
\hline \multirow{4}{*}{ Mimosaseae } & Prosopis juliflora $(\mathrm{Sw}) \mathrm{DC}$ & $*$ & $*$ & \\
\hline & Faidherbia albida (Del.) A. Chev. & & $*$ & \\
\hline & Acacia senegal (L.) Willd & & $*$ & $*$ \\
\hline & Acacia raddiana Savi. & $*$ & $*$ & $*$ \\
\hline Burseraceae & Commiphora africana (A.Rich.) Engl & & $*$ & \\
\hline Moraceae & Ficus sycomorus $\mathrm{L}$ & & $*$ & \\
\hline \multirow{2}{*}{ Arecaceae } & Hyphaene thebaica (L) Mart & & $*$ & \\
\hline & Phoenix dactylifera L & & $*$ & \\
\hline Salvadoraceae & Salvadora persica $\mathrm{L}$ & $*$ & $*$ & $*$ \\
\hline
\end{tabular}

Tableau 2 : Principales caractéristiques structurales des peuplements ligneux en fonction des sites.

\begin{tabular}{lcccc}
\hline Paramètres structuraux & Peuplement & Lac & Kanem & Bahr El Ghazal \\
\hline Densité réelle (ind.ha $\left.{ }^{-1}\right)$ & $29,01 \pm 7,02$ & $34,67 \pm 5,44$ & $32 \pm 6,22$ & $21,39 \pm 3,02$ \\
Densité théorique $\left(\right.$ ind.ha $\left.^{-1}\right)$ & $62,22 \pm 11,49$ & $106,06 \pm 10,19$ & $78,17 \pm 10,72$ & $29,89 \pm 12,52$ \\
Surface terrière $\left(\mathrm{m}^{2} \cdot \mathrm{ha}^{-1}\right)$ & $2,49 \pm 0,01$ & $2,27 \pm 0,02$ & $3,39 \pm 0,03$ & $1,21 \pm 0,01$ \\
Recouvrement aérien $\left(\mathrm{m}^{2} \cdot \mathrm{ha}^{-1}\right)$ & $1160,81 \pm 4,87$ & $1604,30 \pm 159,41$ & $1457,12 \pm 61,94$ & $465,66 \pm 24,53$ \\
\hline
\end{tabular}

\section{DISCUSSION}

Les résultats de cette étude fournissent les premières informations sur l'état actuel de la végétation ligneuse sur le tracé de la GMV au Tchad. La caractérisation du peuplement ligneux de la zone d'étude a révélé 18 espèces, 15 genres et 9 familles. Les familles les plus représentées sont les Mimosaseae. Cependant, nos résultats ne sont pas similaires à ceux de Goy (2012) dans la zone sahélo-soudanienne du Tchad et
Boubacar (2010) dans les zones SahéloSahariens du Niger qui ont trouvé une richesse floristique élevée (respectivement 44 et 23 espèces). Comparé à ces différents résultats, il est apparu que la zone d'étude présente une diversité floristique moyenne (18 espèces). Le nombre d'individus et d'espèces rencontrées varie en fonction des sites. Globalement, six espèces sont communes aux 3 sites, il s'agit de Balanites aegyptiaca, Capparis decidua, Leptadenia 
pyrotechnica, Leptadenia arborea, Acacia raddiana et Salvadora persica. Toutefois, le Kanem se différencie nettement des autres sites par une plus grande richesse floristique et par un plus grand nombre d'espèces exclusives. Cette richesse floristique élevée au Kanem révèle d'une situation climatique plus favorable et d'une pression anthropique moins accentuée. Par contre, le Lac présente une diversité floristique faible, avec une seule espèce exclusive (Calotropis procera). La richesse floristique moyenne au Lac situé dans une dépression dunaire serait due à la disponibilité hydrique liée à la topographie et à l'altitude. L'absence d'espèces exclusives $\mathrm{du}$ Bahr El Ghazal traduit par une richesse floristique moyenne. Les espèces les plus abondantes sont Acacia raddiana et Balanites aegyptiaca. Les espèces rares ou très faiblement représentées sont Calotropis procera, Acacia senegal, Ziziphus spina christi, Leptadenia arborea et Boscia senegalensis. Les densités théorique et réelle ont montré une nette différence entre les sites. La densité théorique au Lac est 3 fois plus importante que la densité réelle, au Kanem, la densité théorique est 2 fois plus importante que la densité réelle et au Bahr El Ghazal, ces deux densités sont presque similaires. Le rapport entre la densité théorique et la densité réelle est plus élevé au Lac et au Kanem, ce qui traduit une distribution en agrégats d'individus. Le contraire est enregistré au Bahr El Ghazal, traduit une distribution régulière (Canard et Poinsot, 2004). La surface terrière est plus élevée au Kanem, moins élevée au Lac et faible au Bahr EL Ghazal. L'importance de cette surface terrière au Kanem s'explique par la forte densité de certaines espèces (Acacia raddiana et Balanites aegyptiaca) et par la présence des arbres à gros troncs (Faidherbia albida, Ficus sycomorus, etc.). Le Lac présente une faible surface terrière liée au fait que cette zone est mise en profit pour les pâturages des troupeaux et des prélèvements de bois à usage divers (Niang et al., 2014; Ndiaye et al., 2014. Au Bahr El
Ghazal, la surface terrière est très faible, ce qui s'explique par la dominance d'arbustes (Boscia senegalensis et Hyphaene thebaica). Le recouvrement élevé au Lac s'explique par la présence de grands arbres à cimes jointives. Au Kanem, le recouvrement est moyen. Cette baisse du recouvrement observée au Kanem s'accentue au Bahr El ghazal à cause des activités anthropiques (Mahamat-Saleh et al., 2013). En ce qui concerne la distribution des arbres par classe de hauteur, la majorité des individus se rencontrent dans les petites classes. Ces données révèlent l'importance de la strate arbustive dans les trois sites, nos résultats sont identiques à ceux de Diallo et al. (2011) dans la zone sahélienne du Sénégal. La dominance des individus observée serait due à l'adaptation progressive des espèces (Acacia raddiana et Balanites aegyptiaca) au milieu. Les gros arbres sont peu fréquents. Quant à la distribution par classe de circonférence, les individus à petits et moyens troncs sont fortement représentés et constituant une savane arbustive. Nos résultats s'accordent aussi avec ceux de Ngaryo et al. (2010), réalisées dans la même zone. Les arbres à hauteur et à grosseur importante sont Faidherbia albida, Ziziphus abyssinica et Ficus sycomorus. Parmi les espèces ayant présenté un nombre élevé de jeunes plants, Acacia raddiana et Balanites aegyptiaca étaient les seules espèces qui semblent occuper davantage l'espace. La forte contribution de ces deux espèces à la régénération du peuplement ligneux reflète la faible pluviométrie de cette zone qui favorise l'émergence d'espèces adaptées aux zones arides. En dehors de ces deux espèces, le potentiel de régénération est faible pour les autres espèces présentes. Les conditions du milieu et le surpâturage semblent être les causes de cette faible régénération (Sarr et al., 2013).

\section{Conclusion}

Ce travail a permis de mettre en évidence la structure et le potentiel de 
régénération des peuplements ligneux des trois sites du tracé de la GMV du Tchad.

Mais présence massive des espèces comme Acacia raddiana et Balanites aegyptiaca ainsi que leur taux de renouvellement très important, attestant de leur adaptation aux conditions pédoclimatiques et aux actions anthropiques dans cette zone, par conséquent, elles doivent être privilégiées dans les programmes de restauration de ces écosystèmes.

Ainsi une connaissance des caractéristiques de la végétation constitue en effet un volet important pour la conception de stratégies de mise en valeur des sites du tracé de la GMV du Tchad.

\section{CONFLIT D'INTERET}

Les auteurs déclarent qu'il n'existe aucun conflit d'intérêt pour cet article.

\section{CONTRIBUTIONS DES AUTEURS}

MM-S a fait le travail de terrain et a rédigé le manuscrit; $\mathrm{ON}, \mathrm{MDD}, \mathrm{KN}$ et $\mathrm{AD}$ ont contribué à la rédaction du manuscrit ; SG a contribué à la collecte des données ; AG a supervisé le travail et a contribué à la correction du manuscrit.

\section{REFERENCES}

ASECNA. 2012. Agence pour la Sécurité de la Navigation Aérienne en Afrique et à Madagascar. Paramètres météorologiques relatifs à la période 2011-2012. Ndjamena-Tchad. Fiche de relevés climatiques, $12 \mathrm{p}$.

Boubacar H. 2010. Caractérisation biophysique des ressources ligneuses dans les zones dégradées et reverdies au Sahel: cas du département de Mayahi. Option «Protection et Restauration des Ecosystèmes Soudaniens et Sahélo-Sahariens». Mémoire de DEA, Université Abdou Moumouni Niamey, Niger, 69 p.

Canard A, Poinsot D. 2004. Quelques méthodes statistiques typiques de l'étude des populations et des peuplements par la méthode des quadrats. Fiche technique. Université de Renne 1, 34 p.

Diallo A, Madiara NF, Guissé A. 2011. Structure des peuplements ligneux dans les plantations d'Acacia sénégal (L.) Willd dans la zone de Dahra (Ferlo, Sénégal). Rev. Écol. (Terre Vie), 66: 415 - 427. DOI : http://irevues.inist.fr/

Floret CH, Pontanier R, Serpantie G. 1993. La Jachère en Afrique Tropicale. Unesco, Dossier Mab16 : Paris, 86 p.

Goy S. 2012. Etude phytosociologique et diagnostic faunique du parc national de manda (TCHAD). Eléments pour un aménagement. Spécialité : écologie, agroforesterie et adaptations. Mémoire de thèse, Université Cheikh Anta Diop, Dakar, 162 p.

Grande Muraille Verte. 2009. Colloque scientifique international sur le choix des espèces végétales et des systèmes de mise en valeur et de suivi, $47 \mathrm{p}$.

Mahamat-Saleh M, Temoua D, Goy S, Goalbaye T. 2012. Compte rendu de la mission de terrain relatif aux travaux de recherche doctorale sur le tracé de la Grande Muraille Verte au Tchad. 18 p.

Mahamat-Saleh M, Diallo A, Ndiaye O, Madiara NF, Guissé A. 2013. Caractérisation des peuplements ligneux de la zone Cayor Baol (ThièsSénégal). Int. J. Biol. Chem. Sci. 7(5): 2117-2132, DOI: http://ajol.info/ index.php/ijbcs

Ndiaye O, Diallo A, Stephen AW, Guissé A. 2014. Structural Diversity of Woody Species in the Senegalese Semi-Arid Zone-Ferlo. American Journal of Plant Sciences, 5: 416-426. DOI: http://dx.doi.org/10.4236/ajps,

Ngaryo FT, Goudiaby VC, Akpo LE. 2010. Caractéristiques d'une gommeraie d'Acacia senegal (L.) Wild. dans la région du Chari Baguirmi au Tchad. Journal des Sciences, 10(2): 13 - 23. DOI : http://www.cadjds.org 
Niang K, Ndiaye O, Diallo A, Guissé A. 2014. Flore et structure de la végétation ligneuse le long de la Grande Muraille Verte au Ferlo, nord Sénégal. Journal of Applied Biosciences, 79: 6938 - 6946. DOI: http://dx.doi.org/10.4314/jab.v79i1.15

Pias J. 1970. La Végétation du Tchad, ses Rapports Avec le Sol. Variations Paléoclimatiques au Quaternaire. ORSTOM : Paris.

Sarr O, Bakhoum A, Diatta S, Akpo LE. 2013. L'arbre en milieu soudanosahélien dans le bassin arachidier
(Centre-Sénégal). Journal of Applied Biosciences 61: 4515 - 4529. 15 p. DOI:

http://dx.doi.org/10.4314/jab.v79i1.15

SNPA/GMV. 2012. Stratégie nationale et plan d'actions pour la mise en œuvre de l'initiative grande muraille verte au Tchad. Ministère de l'environnement et des ressources halieutiques. 70 p.

Wouters P, Notelaers V. 1999. L'espace entre les arbres et la densité des peuplements. Sylva Belgica, 106(21): 4. 